\title{
ON THE RANK, ORDER AND CLASS OF ALGEBRAIC MINIMUM CURVES*
}

BY

\section{ARTHUR SULLIVAN GALE}

The equations of all analytic minimum curves may be written in terms of a complex parameter $s$ and an arbitrary analytic function $F(s)$. The curve will be algebraic when and only when $F(s)$ is an algebraic function, and then the rank, order and class of the curve can be easily expressed as the orders of three functions, $\psi(s), \phi(s)$ and $\chi(s),($ see $\S 1)$, of the general form

$$
f\left[s, F(s), F^{\prime}(s), F^{\prime \prime}(s)\right] \text {. }
$$

It is the purpose of this paper to give the developments in series of

$$
\psi(s), \phi(s) \text { and } \chi(s)
$$

from which the rank, order and class of the minimum curve corresponding to $F(s)$ may be determined if the developments of $F(s)$ be known in the vicinity of its poles, branch points and the point at infinity. Detailed application is then made to the case in which $F(s)$ is a two-valued function of its argument, the case in which $F(s)$ is a rational function having been treated by LIE. $\dagger$ Finally attention is called to the relation of this work to the theory of minimum surfaces.

\section{$\S 1$. Equations and properties of minimum curves. $\ddagger$}

All minimum curves, except minimum lines, may be represented in the form

$$
\Gamma:\left\{\begin{array}{l}
x=\left(1-s^{2}\right) F^{\prime \prime}(s)+2 s F^{\prime}(s)-2 F(s) \\
y=i\left(1+s^{2}\right) F^{\prime \prime}(s)-2 i s F^{\prime}(s)+2 i F(s) \\
z=2 s F^{\prime \prime}(s)-2 F^{\prime}(s)
\end{array}\right.
$$

where $F(s)$ is an analytic function subject to the sole condition that $F^{\prime \prime \prime}(s) \neq 0$; $\Gamma$ is an algebraic curve when and only when $F(s)$ is an algebraic function; its osculating plane is

$$
\left(1-s^{2}\right) x+i\left(1+s^{2}\right) y+2 s z+4 F(s)=0 .
$$

* Presented to the Society February 22, 1902. Received for publication August 13, 1902.

† Mathematische Annalen, vol. 14 (1879), pp. 368, 371, 372.

$\ddagger$ These properties are to be found in essentially the same form in DARBoux, Théorie des Surfaces, §§ 235, 198. 
Theorem. The order of $\Gamma$ is the order of the function $\phi(s)=A\left[\left(1-s^{2}\right) F^{\prime \prime}(s)+2 s F^{\prime}(s)-2 F(s)\right]$ $+i B\left[\left(1+s^{2}\right) F^{\prime \prime}(s)-2 s F^{\prime}(s)+2 F(s)\right]+2 C\left[s F^{\prime \prime}(s)-F^{\prime}(s)\right]+D$.

For to every value $s=s_{0}$ such that $\phi\left(s_{0}\right)=0$ corresponds a point of intersection of $\Gamma$ with the plane of general position

$$
A x+B y+C z+D=0 .
$$

Theonem. The rank of $\Gamma$ is the order of

$$
\begin{array}{r}
\psi(s)=2 A\left[\left(1-s^{2}\right) F^{\prime}(s)+2 s F(s)\right]+2 i B\left[\left(1+s^{2}\right) F^{\prime}(s)-2 s F^{\prime}(s)\right] \\
+4 C\left[s F^{\prime}(s)-F(s)\right]+i A_{1}\left(1-s^{2}\right)-B_{1}\left(1+s^{2}\right)+2 i C_{1} s .
\end{array}
$$

For the vanishing of $\psi(s)$ is the condition that the line of general position

will intersect a generator

$$
\begin{aligned}
& B z-C y=A_{1} \\
& C x-A z=B_{1} \\
& A y-B x=C_{1}
\end{aligned} \quad A A_{1}+B B_{1}+C C_{1}=0
$$

$$
\begin{gathered}
\left(1-s^{2}\right) x+i\left(1+s^{2}\right) y+2 s z+4 F(s)=0 \\
-s x+i s y+z+2 F^{\prime}(s)=0
\end{gathered}
$$

of the envelope of (1).

Theonem. The class of $\Gamma$ is the order of

$$
\chi(s)=\left(1-s^{2}\right) A+i\left(1+s^{2}\right) B+2 s C+4 F(s) .
$$

For the condition that the plane (1) shall pass through the point $(A, B, C)$ is that $\chi(s)=0$.

TheOREM. If $\Gamma$ be subjected to a displacement the transformed curve is given by the equations

where

$$
\begin{aligned}
& x^{\prime}=\left(1-t^{2}\right) G^{\prime \prime}(t)+2 t G^{\prime}(t)-2 G(t) \\
& y^{\prime}=i\left(1+t^{2}\right) G^{\prime \prime}(t)-2 i t G^{\prime}(t)+2 i G(t) \\
& z^{\prime}=2 t G^{\prime \prime}(t)-2 G^{\prime}(t)
\end{aligned}
$$

(a) $\quad t=s, \quad G(t)=F(s)-\frac{\lambda}{4}\left(1-s^{2}\right)-\frac{i \mu}{4}\left(1+s^{2}\right)-\frac{\nu}{2} s$

if the displacement be a translation whose components parallel to the axes are $\lambda, \mu, \nu ;$ or

$$
\begin{gathered}
t=\frac{m_{0} s-n}{n_{0} s+m}, \quad \delta=m m_{0}+n n_{0}, \\
G(t)=\frac{\left(m_{0}-n_{0} t\right)^{2}}{\delta} F\left(\frac{m t+n}{m_{0}-n_{0} t}\right)
\end{gathered}
$$


if the displacement be a rotation about the origin through the same angle as the complex sphere is rotated by the transformation

$$
t=\frac{m_{0} s-n}{n_{0} s+m} .
$$

The first of these transformations shows that if

$$
F(s)=f(s)+\alpha s^{2}+\beta s+\gamma
$$

it may be reduced to $f(s)$ by translating $\Gamma$ through a distance whose components are $2(\gamma-\alpha),-2 i(\gamma+\alpha), 2 \beta$. The second transformation shows that by properly orienting the curve $\Gamma$ the function $F(s)$ may be chosen in such a way that $s=\infty$ will not be one of its branch points. We will always suppose that $\Gamma$ is oriented in this manner.

\section{§ 2. Development of the functions $\phi(s), \psi(s)$ and $\chi(s)$.}

When $F(s)$ is an algebraic function it may be developed in the vicinity of any point of the plane, $s=a$, in one of the forms

$$
\begin{gathered}
F(s)=(s-a)^{\frac{p}{q}} P(s-a)^{\frac{1}{q}}, \\
F(s)=(s-a)^{p} P(s-a), \\
F(s)=\left(\frac{1}{s}\right)^{p} P\left(\frac{1}{s}\right) \\
F(s)=\left(\frac{1}{s}\right)^{\frac{p}{q}} P\left(\frac{1}{s}\right)^{q}
\end{gathered}
$$

where $p$ and $q$ are integers and $P(x)$ denotes a series of the form

$$
P(x)=\alpha_{0}+\alpha_{1} x+\alpha_{2} x^{2}+\cdots, \bar{j}
$$

in which $\alpha_{0} \neq 0$. After the convention of the preceding section we need not consider developments of the form (7). Assuming $F(s)$ to be successively of the forms (4), (5) and (6) we shall determine the corresponding developments of $\phi(s), \psi(s)$ and $\chi(s)$ about $s=a$; we shall then be able to determine the various types of points which are poles of these functions and if we can determine all points of $F(s)$ of the types found we can then determine the orders of $\phi(s), \psi(s)$ and $\chi(s)$ by summing the orders of their poles. The developments of this section are applicable to any algebraic minimum curve corresponding to a given function $F(s)$ and hence may be applied to the determination of the order, rank and class of that curve. 
Consider $\psi(s)$ first. If we suppose that $F(s)$ has the form (4) I find that $\psi(s)$ will have the form,

$$
\begin{gathered}
\psi(s)=2(s-a)^{\frac{p-q}{q}}\left\{\beta \alpha_{0} \frac{p}{q}+\beta \alpha_{1} \frac{p+1}{q}(s-a)^{\frac{1}{q}}+\beta \alpha_{2} \frac{p+2}{q}(s-a)^{\frac{2}{q}}+\cdots\right. \\
+\left[\beta \alpha_{q} \frac{p+q}{q}+\beta^{\prime} \alpha_{0} \frac{p-q}{q}\right](s-a)+\left[\beta a_{q+1} \frac{p+q+1}{q}\right. \\
\left.\left.+\beta^{\prime} \alpha_{1} \frac{p-q+1}{q}\right](s-a)^{q+1}+\cdots\right\}+i\left\{\beta_{1}+\beta_{1}^{\prime}(s-a)+\frac{\beta_{1}^{\prime \prime}}{2}(s-a)^{2}\right\}
\end{gathered}
$$

where

$$
\begin{aligned}
& \beta=A\left(1-a^{2}\right)+i B\left(1+a^{2}\right)+2 C a, \\
& \beta_{1}=A_{1}\left(1-a^{2}\right)+i B_{1}\left(1+a^{2}\right)+2 C_{1} a,
\end{aligned}
$$

and primes denote differentiation with respect to $a$. Notice that we can always suppose $\beta \neq 0$, as otherwise the line (3) would not be a line of general position but would belong to the linear complex defined by $\beta=0$. Now $s=a$ can be a pole of $\psi(s)$ only when $p<q$. If $0<p<q, s=a$ will be a pole of order $q-p$; while if $p=0$, the order of the pole will be $q-i$ if

$$
\alpha_{1}=\alpha_{2}=\cdots=\alpha_{i-1}=0, \quad \alpha_{i} \neq 0, \quad i<q,
$$

but the point will not be a pole if $i \geqq q$. When $p<0$ the order of the pole at $s=a$ is $|p|+q$.

If $F(s)$ has the form (5) we have only to interpret the above results for $q=1$. Hence in this case $s=a$ will be a pole of $\psi(s)$ only when $p<0$ and then the order of the pole is $|p|+1$.

Finally, in the vicinity of $s=\infty$, if $F(s)$ has the form (6) I find that

$$
\begin{aligned}
\psi(s)=2\left(\begin{array}{c}
1 \\
s
\end{array}\right)^{p-1}\left\{\gamma \alpha_{0}(p+2)+\right. & {\left[\gamma \alpha_{1}(p+3)-2 C \alpha_{0}(p+1)\right]\left(\frac{1}{s}\right) } \\
\left.+\left[\gamma \alpha_{2}(p+4)-\delta \alpha_{0} p-2 C \alpha_{1}(p+2)\right]\left(\frac{1}{s}\right)^{2}+\cdots\right\} & +i\left(\frac{1}{s}\right)^{-2}\left\{-\gamma_{1}+2 C_{1}\left(\frac{1}{s}\right)+\delta_{1}\left(\frac{1}{s}\right)^{2}\right\},
\end{aligned}
$$

where

$$
\begin{aligned}
\gamma=A-i B, & \delta=A+i B, \\
\gamma_{1}=A_{1}-i B_{1}, & \delta_{1}=A_{1}+i B_{1} .
\end{aligned}
$$

When $p \geqq 0, s=\infty$ will be a pole of $\psi(s)$ of order 2 , for we can suppose that $\gamma_{1} \neq 0$. When $p=-1$ or -2 the order of the pole is still 2 , for we can further suppose in the first case that $2 \gamma x_{0}-i \gamma_{1} \neq 0$ or, in the second case, that $2 \gamma \alpha_{1}+4 C \alpha_{0}-i \gamma_{1} \neq 0$. Hence, when $p \geqq-2$ we see that $s=\infty$ is a pole of $\psi(s)$ of order 2 and when $\gamma<-2$ it will be a pole of order $|p|+1$. 
Consider now the corresponding developments of $\phi(s){ }^{*}$ When $F(s)$ has the form (4) I find that

$$
\begin{aligned}
\phi(s)=D & +(s-a)^{\frac{p-2 q}{q}}\left\{\beta \alpha_{0} \frac{p}{q} \frac{p-q}{q}+\beta \alpha_{1} \frac{p+1}{q} \frac{p-q+1}{q}(s-a)^{\frac{1}{q}}\right. \\
& +\beta \alpha_{2} \frac{p+2}{q} \frac{p-q+2}{q}(s-a)^{\frac{2}{q}}+\cdots+\left[\beta \alpha_{q} \frac{p+q}{q} \frac{p}{q}\right. \\
& \left.+\beta^{\prime} \frac{p}{q} \frac{p-2 q}{q} \alpha_{0}\right](s-a)+\left[\beta \alpha_{q+1} \frac{p+q+1}{q}\right. \\
& \left.\left.+\beta^{\prime} \alpha_{1} \frac{p+1}{q} \frac{p-2 q+1}{q}\right](s-a)^{\frac{q+1}{q}}+\cdots\right\},
\end{aligned}
$$

where $\beta$ and $\beta^{\prime}$ have the same significance as above. Then $s=a$ cannot be a pole of $\phi(s)$ unless $p<2 q$. When $q<p<2 q$ it is a pole of order $2 q-p$; while if $p=q$ the order of the pole is $q-i$ if

$$
\alpha_{1}=\alpha_{2}=\cdots=\alpha_{i-1}=0, \quad \alpha_{i} \neq 0, \quad i<q ;
$$

but if $i \geqq q$ then $s=a$ will not be a pole of $\phi(s)$. Likewise, if $0<p<q$ the order of the pole at $s=a$ is $2 q-p$; when $p=0$ the pole is of order $2 q-j$ if

or if

$$
\alpha_{1}=\alpha_{2}=\cdots=\alpha_{j-1}=0, \quad \alpha_{j} \neq 0, \quad j<q,
$$

$$
\begin{gathered}
\alpha_{1}=\alpha_{2}=\cdots=\alpha_{q-1}=0, \quad \alpha_{q} \gtreqless 0, \quad \alpha_{q+1}=\alpha_{q+2}=\cdots=\alpha_{j-1}=0, \\
\alpha_{j} \neq 0, \quad q<j<2 q ;
\end{gathered}
$$

but if $j \geqq 2 q$ then $s=a$ will cease to be a pole of $\phi(s)$. Finally, if $p<0$ there will always be a pole at $s=a$ of order $|p|+2 q$.

Interpreting these results for $q=1$ we will have the development of $\phi(s)$ derived from the form (5) of $F(s)$. In this case $s=a$ will be a pole of $\phi(s)$ only when $p<0$; for if $p=1$ the first coefficient, and if $p=0$ the first two coefficients of the series will vanish. When $p<0$ there will be a pole of $\phi(s)$ at $s=a$ of order $|p|+2$.

Finally, if $F(s)$ is of the form (6) I find that

$$
\begin{aligned}
& \phi(s)=D+\left(\frac{1}{s}\right)^{p}\left\{-\gamma \alpha_{0}(p+1)(p+2)\right. \\
& +\left[-\gamma \alpha_{1}(p+2)(p+3)+2 C \alpha_{0} p(p+2)\right]\left(\begin{array}{l}
1 \\
s
\end{array}\right) \\
& \left.+\left[-\gamma \alpha_{2}(p+3)(p+4)+\delta \alpha_{0} p(p+1)+2 C \alpha_{1}(p+1)(p+2)\right]\left(\frac{1}{s}\right)^{2}+\cdots\right\} \\
& \text { where } \quad \gamma=A-i B \text { and } \delta=A+i B .
\end{aligned}
$$

* As the general values of the constants $A, B$ and $C$ will prevent the poles of the coefficients of $A, B$ and $C$ from destroying each other, it follows that the infinite points of $\Gamma$ will be derived trom those values of $s$ giving rise to poles of $\phi(s)$. 
As in the preceding case, $s=\infty$ cannot be a pole of $\phi(s)$ unless $p<-2$; for if $p=-1$ or -2 the coefficients of the powers of $1 / s$ with negative exponents will vanish. Hence $s=\infty$ is a pole of $\phi(s)$ only when $p<-2$ in which case it is a pole of order $|p|$.

The various developments of $\chi(s)$ can be so easily written down that only the conclusions drawn from those developments need be stated. When $F(s)$ is of the form (4) or (5) $s=a$ will be a pole of $\chi(s)$ when and only when $p<0$, the order of the pole being $|p|$ in each case. But if $F(s)$ has the form (6) $s=\infty$ will be a pole of $\chi(s)$ of order 2 unless $p<-2$ when the order is $|p|$.

Thus we see that the only values of $s$ which can be poles of $\phi(s), \psi(s)$ or $\chi(s)$ are the poles and branch points of $F(s)$ and the point at infinity. When the developments of $F(s)$ about these points are known the order, rank and class of the minimum curve corresponding to $F(s)$ can be easily determined.

\section{§3. Application to the case in which $F(s)$ is a rational function.*}

For completeness we will apply the results of the preceding section to the case which LIE considered from a less general standpoint. The application is simplified by a suitable choice of axes. Notice first, however, that if a rational function of $s$ be subjected to a transformation of the form

$$
s=\frac{\alpha s^{\prime}+\beta}{\gamma s^{\prime}+\delta}
$$

that the transformed function is a rational function in which the degree of the numerator equals that of the denominator. Hence by rotating the minimum curve $\Gamma$ we can always reduce the rational function $F(s)$ to a rational function the degree of whose numerator exceeds that of the denominator by 2 ; and then by applying an appropriate translation to $\Gamma$ we can reduce $F^{\prime}(s)$ to a rational function whose numerator is of lower degree than its denominator. $F(s)$ has no branch points and its only poles are now at the zeros of the denominator of which we will suppose there are $m$ of orders $m_{1}, m_{2}, \cdots, m_{m}$ respectively.

The results of $\S 2$ show that the order of $\psi(s)$, i. e., the rank of $\Gamma$ is

$$
R=\sum_{1}^{m} m_{i}+m+2 ;
$$

that the order of $\phi(s)$, which gives the order of $\Gamma$, is

$$
O=\sum_{1}^{m} m_{i}+2 m,
$$

\footnotetext{
* In geometrical language, the osculating plane of $\Gamma$ envelops a developable containing the imaginary circle at infinity as a single line. In general, the circle at infinity will be an $n$-fold line on that developable if $F(s)$ is $n$-valued. Compare DAkBovx, l. c., §235.
} 
and that the class of $\Gamma$, or the order of $\chi(s)$, is

$$
C=\sum_{1}^{m} m_{i}+2 \text {. }
$$

The simplest of these curves corresponds to the hypothesis $m=m_{1}=1$ or $F(s)=1 /(s-a)$. The rank of $\Gamma$ is 4 , its order 3 and its class 3 . Numerous other examples are given by LIE in $\S 11$ of the paper already cited. In the following sections we will extend LIE's work to the case in which $F(s)$ is two-valued.

§4. Normal form of $F(s)$ when $F(s)$ is two-valued.

The application of the results of $\S 2$ to the case in which $F(s)$ is a twovalued function is materially simplified by a suitable orientation of the curve $\Gamma$. When $F(s)$ is two-valued it may be written in the form*

$$
F(s)=R_{1}(s)+R_{2}(s) \sqrt{P(s)},
$$

where $R_{1}(s)$ and $R_{2}(s)$ are rational functions and $P(s)$ a polynomial without multiple roots of degree $\lambda$. By rotating $\Gamma$ about the origin $F(s)$ may be thrown into the form

$$
\begin{aligned}
F(s) & =\frac{\left(m_{0}-n_{0} s\right)^{2}}{\delta}\left[\frac{P_{1}(s)}{P_{2}(s)}+\frac{P_{3}(s)}{P_{4}(s)} \frac{\sqrt{\prod_{1}^{\lambda}\left(s-e_{i}\right)}}{\left(m_{0}-n_{0} s\right)^{\lambda / 2}}\right] \\
= & \frac{P_{1} P_{4}\left(m_{0}-n_{0} s\right)^{\frac{\lambda}{2}}+P_{2} P_{3} \sqrt{\prod_{1}^{\lambda}\left(s-e_{i}\right)}}{\delta P_{2} P_{4}\left(m_{0}-n_{0} s\right)^{(\lambda-4) / 2}}
\end{aligned}
$$

where the $P_{i}$ are polynomials such that the degrees of $P_{1}$ and $P_{3}$ are equal, respectively, to those of $P_{2}$ and $P_{4}$. When $\lambda$ is even we may set $\lambda=2 p+2$ and when odd we may rationalize the denominator and set $\lambda+1=2 p+2$. Thus, in either case, if we neglect all factors common to the numerator and denominator and also neglect any constant factor, the effect of which will be to replace the given minimum curve by one similar to it, we may always write $F(s)$ in the form

where

$$
F(s)=\frac{C \prod_{1}^{l}\left(s-a_{i}\right)^{l_{i}}+\prod_{1}^{m}\left(s-b_{i}\right)^{m_{i}} \sqrt{\prod_{1}^{2 p+2}\left(s-e_{i}\right)}}{\prod_{1}^{n}\left(s-c_{i}\right)^{n_{i}}}
$$

$$
\sum_{1}^{l} l_{i}=\sum_{1}^{n} n_{i}+2
$$

* Compare Lre, Archiv for Mathematik og Naturvidenskab, vol. 2 (1877), p. 195. 


$$
\sum_{1}^{n} n_{i}=\sum_{1}^{m} m_{i}+p-1
$$

By a suitable translation of $\Gamma$ the first of these conditions may be replaced by the condition

$$
\sum_{1}^{l} l_{i}<\sum_{1}^{n} n_{i}
$$

In the following sections we will suppose that $F(s)$ has the form (8) with the conditions

$$
\sum_{1}^{l} l_{i}<\sum_{1}^{n} n_{i}=\sum_{1}^{m} m_{i}+p-1
$$

Thus if $p$ and $\sum m_{i}$ are given the value of $\sum n_{i}$ is determined and that of $\sum l_{i}$ is known as fully as proves necessary for our purposes. As the transformations to which $F(s)$ has been subjected do not introduce any negative exponents the case $\sum m_{i}=p=0$, and hence $\sum n_{i}=-1$, is to be excluded although it is easily seen to be identical with the case $\sum n_{i}=p=0, \sum m_{i}=1$.

\section{§5. Development of $F(s)$ about its poles, branch points and the point at} infinity.

We have now to consider the various possible developments of (8) in the vicinity of those points which may be poles of any of the three functions whose orders we wish to compute. At first let the points $a_{i}, b_{i}, c_{i}$ and $e_{i}$ be all distinct. It is unnecessary to consider the developments of $F(s)$ about $s=a_{i}$ and $s=b_{i}$.

About any branch point $s=e_{i}$ we have

where certainly

$$
F(s)=\alpha_{0}+\alpha_{1}\left(s-e_{i}\right)^{3 / 2}+\alpha_{2}\left(s-e_{i}\right)+\cdots
$$

$$
\alpha_{0}, \alpha_{1} \neq 0 \text {. }
$$

In the vicinity of $s=\infty$ we have

$$
\begin{aligned}
F(s)= & C s^{\Sigma I_{i}} \prod_{1}^{l}\left(1-\frac{a_{i}}{s}\right)^{l_{i}}+s^{\Sigma m_{i}+p+1} \prod_{1}^{m}\left(1-\frac{b_{i}}{s}\right)^{m_{i}} \sqrt{\prod_{1}^{2 p+2}\left(1-\frac{e_{i}}{s}\right)} \\
s^{\Sigma n_{i}} \prod_{1}^{n}\left(1-\frac{c_{i}}{s}\right)^{n_{i}} & \left(\frac{1}{s}\right)^{-2}\left\{\alpha_{0}+\alpha_{1}\left(\frac{1}{s}\right)+\cdots\right\}, \quad \alpha_{0} \neq 0
\end{aligned}
$$

since from (9)

$$
\sum m_{i}+p+1=\sum n_{i}+2>\sum l_{i}
$$

and the development commences with terms of the same order in each sheet of the Riemann's surface of two sheets on which $F(s)$ is rational. 
In the vicinity of $s=c_{i}$ we have

$$
F(s)=\left(s-c_{i}\right)^{-n_{i}}\left\{\alpha_{0}+\alpha_{1}\left(s-c_{i}\right)+\cdots\right\}, \quad \alpha_{0} \neq 0
$$

and the exponent of the first factor will be the same for each sheet of the Rieman'n's surface. It may happen, however, that the developments of

$$
P=C \prod_{1}^{l}\left(s-a_{i}\right)^{l_{i}} \quad \text { and } \quad Q=\prod_{1}^{m}\left(s-b_{i}\right)^{m_{i}} \sqrt{\prod_{1}^{2 p+2}\left(s-e_{i}\right)}
$$

in the vicinity of some of these points, say the $r$ points $c_{i}, i=n-r+1, \ldots, n$, may be the same for a number of terms. Then in one sheet, say the upper one, the order of the pole of $F(s)$ at $c_{i}$ will be less than $n_{i}$, and, in fact, the point may cease to be a pole in that sheet. In order that the first $r_{i}+1$ terms of these developments be identical it is necessary and sufficient that

$$
\left\{\begin{array}{c}
{[P]_{s=c_{i}}=[Q]_{s=c_{i}},} \\
\frac{d}{d s}[P]_{s=c_{i}}=\frac{d}{d s}[Q]_{s=c_{i}}, \\
\frac{d^{r_{i}}}{d s^{r_{i}}}[P]_{s=c_{i}}=\frac{d^{r_{i}}}{d s^{r_{i}}}[Q]_{s=c_{i}} .
\end{array}\right.
$$

When these equations are satisfied $F(s)$ will have the form (10) about $s=c_{i}$ when $s$ is in the lower sheet; but in the upper sheet its development will be of the form

$$
F(s)=\left(s-c_{i}\right)^{-n_{i}+r_{i}+1}\left\{\alpha_{0}+\alpha_{1}\left(s-c_{i}\right)+\cdots\right\}, \quad \alpha_{0} \neq 0 .
$$

We need never consider $r_{i}>n_{i}-1$ as $r_{i}=n_{i}-1$ is sufficient to make $s=c_{i}$ cease to be a pole of $F(s)$ and hence, also, cease to be a pole of $\phi(s), \psi(s)$ or $\chi(s)$.

It is now necessary to examine all the possible cases in which the points $a_{i}, b_{i}, c_{i}$ and $e_{i}$ are not distinct. If we denote by an equality of the form $a_{i}=b_{i}$ the fact that one of the points $s=a_{i}$ is coincident with some one of the points $8=b_{i}$ the possible cases are :
1) $a_{i}=b_{i}$,
7) $a_{i}=b_{i}=c_{i}$,
2) $a_{i}=c_{i}$,
8) $a_{i}=b_{i}=e_{i}$,
3) $b_{i}=c_{i}$,
9) $a_{i}=c_{i}=e_{i}$,
4) $a_{i}=e_{i}$,
10) $b_{i}=c_{i}=e_{i}$,
5) $b_{i}=e_{i}$
11) $a_{i}=b_{i}=c_{i}=e_{i}$.
6) $c_{i}=e_{i}$, 
Of these we may at once exclude cases 7 and 11 from consideration. Case 1 may be neglected as it will not affect the developments of $F(s)$ in the vicinity of its poles, branch points and the point at infinity. Cases 2 and 3 have no effect on the developments of the form (10) except that condition (11) cannot be satisfied by such points. Cases 6 and 10 will give developments of the same form and so may be treated together. In the remaining cases $F(s)$ will asume developments of distinct forms.

For convenience of reference the developments needed in the following section are given in the accompanying table. The first column characterizes the point about which $F(s)$ has the form given in the second column where $P(x)$ denotes the series

$$
P(x)=\alpha_{0}+\alpha_{1} x+\alpha_{2} x^{2}+\cdots, \quad\left(\alpha_{0} \neq 0\right) .
$$

In the third column are remarks of an essential nature concerning the series in

\begin{tabular}{|c|c|c|}
\hline$e_{i}$ & $P\left(s-e_{i}\right)^{1 / 2}$ & $\alpha_{1} \neq 0$ \\
\hline$c_{i}, i \leqq n-r$ & $\left(s-c_{i}\right)^{-n_{i}} P\left(s-c_{i}\right)$ & in each sheet. \\
\hline$c_{i}, i>n-r$ & $\begin{array}{c}\left(s-c_{i}\right)^{-n_{i}+r_{i}+1} P\left(s-c_{i}\right) \\
\left(s-c_{i}\right)^{-r_{i}} P\left(s-c_{i}\right)\end{array}$ & $\begin{array}{l}\text { in one sheet. } \\
\text { in other sheet. }\end{array}$ \\
\hline$a_{i}=e_{i}$ & $\left(s-e_{i}\right)^{1 / 8} P\left(s-e_{i}\right)^{1 / 3}$ & \\
\hline$b_{i}=e_{i}$ & $P .\left(s-e_{i}\right)^{3 / 2}$ & $\begin{array}{llll} & \alpha_{3} \neq 0 & \text { if } & m_{i}=1 \\
& \alpha_{3}=0 & \text { “ } & m_{i}>1\end{array}$ \\
\hline$c_{i}=e_{i}$ & $\left(s-e_{i}\right)^{-\frac{2 n_{i}}{2}} P\left(s-e_{i}\right)^{1 / 2}$ & \\
\hline$a_{i}=b_{i}=e_{i}$ & $\left(s-e_{i}\right)^{\frac{e}{2}} P\left(s-e_{i}\right)^{1 / 2}$ & $\begin{array}{lll}\epsilon=2, \alpha_{1} \neq 0 & \text { if } & m_{i}=1, l_{i}=1 \\
\epsilon=3 & \text { “ } & m_{i}=1, l_{i}>1 \\
\epsilon=2, \alpha_{1}=0 & \text { “ } & m_{i}>1, l_{i}=1 \\
\epsilon>3 & \text { “ } & m_{i}>1, l_{i}>1\end{array}$ \\
\hline$a_{i}=c_{i}=e_{i}$ & $\left(s-e_{i}\right)^{-\frac{2 n_{i}-1}{2}} P\left(s-e_{i}\right)^{1 / 3}$ & \\
\hline$\infty$ & $\left(\frac{1}{s}\right)^{-2} P\left(\frac{1}{s}\right)$ & in each sheet. \\
\hline
\end{tabular}
the second column. 
§ 6. The rank, order and class of $\Gamma$ when $F(s)$ is two-valued.

The rank, order and class of the minimum curve $\Gamma$ corresponding to the twovalued function $F(s)$ can now be obtained by combining the results of $\S 2$ and $\S 5$. Notice first that the points $a_{i}=e_{i}$ do not need to be distinguished from the points $e_{i}$; for both give rise to simple poles of $\psi(s)$ and poles of $\phi(s)$ of order 3. In like manner the points $\alpha_{i}=b_{i}=e_{i}$ may be counted with the points $b_{i}=e_{i}$ since neither are poles of $\psi(s)$ and they are simple poles of $\phi(s)$ when and only when $m_{i}=1$, ceasing to be poles if $m_{i}>1$. But one further point needs special mention, viz., the $r$ points ( $r$ may be zero) $s=c_{i}$ which satisfy the conditions (11). In one sheet of the Riemann's surface $s=c_{i}$ will be a pole of $\psi(s)$ of order $n_{i}+1$ and of $\phi(s)$ of order $n_{i}+2$; while in the other sheet the order of the pole of $\psi(s)$, if the point is a pole, will be $n_{i}+1-\rho_{i}$ where

$$
\rho_{i}=\left\{\begin{array}{lll}
0 & \text { if conditions }(11) \text { are not satisfied, } \\
r_{i}+1 & \text { “ } & r_{i}<n_{i}-1, \\
n_{i}+1 & \text { “ } & r_{i} \geqq n_{i}-1,
\end{array}\right.
$$

the point ceasing to be a pole when $\rho_{i}$ assumes its maximum value; and the order of the pole $\phi(s)$ at $s=c_{i}$, if the point is a pole, will be $n_{i}+2-\sigma$ where

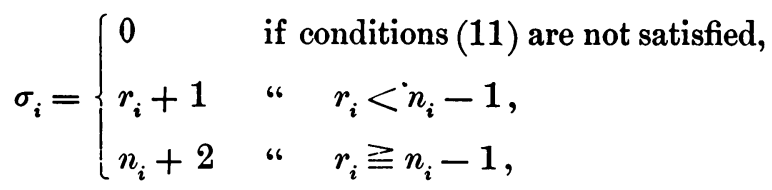

the point ceasing to be a pole when $\sigma_{i}$ takes on its maximum value.

The following table is convenient in summing the orders of $\psi(s)$ and $\phi(s)$. The first column gives the number of points of $F(s)$ of the sort characterized in the second column; the numbers defined in this column are evidently subject to the conditions

$$
\begin{gathered}
\mu+\nu+r \leqq n, \\
\lambda+\mu+\nu \leqq 2 p+2, \\
\lambda_{1}+\lambda_{2}=\lambda .
\end{gathered}
$$

In the third column is given the order of the pole of $\psi(s)$ at the corresponding point in the second column; and in the fourth column in the sum of the orders over all such points. The fifth and sixth columns bear the same relation to $\phi(s)$ as the third and fourth do to $\psi(s)$. In counting the number of points at the poles $s=c_{i}$ and the point at infinity the number is doubled to account for both sheets of the Riemann's surface on which $F(s)$ is rational. 


\begin{tabular}{|c|c|c|c|c|c|}
\hline$\lambda\left\{\begin{array}{l}\lambda_{1}, m_{i}=1 \\
\lambda_{2}, m_{i}>1\end{array}\right.$ & $b_{i}=e_{i}$ & no pole & & $\left\{\begin{array}{c}1 \\
\text { no pole }\end{array}\right.$ & $\lambda_{1}$ \\
\hline$v$ & $c_{i}=e_{i}$ & $2 n_{i}+2$ & $2 \sum_{1}^{\nu} n_{i}+2 v$ & $2 n_{i}+4$ & $2 \sum_{1}^{\nu} n_{i}+4 \nu$ \\
\hline$\mu$ & $a_{i}=c_{i}=e_{i}$ & $2 n_{i}+1$ & $2 \sum_{i+1}^{\nu+\mu} n_{i}+\mu$ & $2 n_{i}+3$ & $2 \sum_{\nu+1}^{\nu+\mu} n_{i}+3 \mu$ \\
\hline $\begin{array}{l}2 p+2 \\
\quad-(\lambda+\mu+v)\end{array}$ & $e_{i}$ & 1 & $2 p+2-\lambda-\mu-v$ & 3 & $\begin{aligned} 6 p+6 & -3 \lambda \\
- & 3 \mu-3 v\end{aligned}$ \\
\hline$\stackrel{2 n}{-2(\mu+\nu+r)}$ & $c_{i}, i \leqq n-r$ & $n_{i}+1$ & $\begin{array}{l}2 \sum_{\nu+\mu+1}^{n-r} n_{i}+2 n \\
-2 \mu-2 \nu-2 r\end{array}$ & $n_{i}+2$ & $\begin{array}{l}2 \sum_{\nu+\mu+1}^{n-r} n_{i}+4 n \\
-4 \mu-4 \nu-4 r\end{array}$ \\
\hline $2 r$ & $c_{i}, i>n-r$ & $\left\{\begin{array}{l}n_{i}+1 \\
n_{i}+1-p_{i}\end{array}\right.$ & $\begin{array}{c}2 \sum_{n=r+1}^{n} n_{i}+2 r \\
-\sum_{n-r+1}^{n} \rho_{i}\end{array}$ & $\left\{\begin{array}{l}n_{i}+2 \\
n_{i}+2-\sigma_{i}\end{array}\right.$ & $\begin{aligned} 2 \sum_{n-r+1}^{n} n_{i} & +4 r \\
& -\sum_{n-r+1}^{n} \sigma_{i}\end{aligned}$ \\
\hline 2 & $\infty$ & 2 & 4 & no pole & \\
\hline
\end{tabular}

Summing the fourth and sixth columns we have the

TheOREM. - The rank of the minimum curve $\Gamma$ corresponding to

is

$$
\begin{gathered}
F(s)=\frac{\left.C \prod_{1}^{l}\left(s-a_{i}\right)^{l_{i}}+\prod_{1}^{m}\left(s-b_{i}\right)^{m_{i}} \sqrt{\prod_{1}^{2 p+2}\left(s-e_{i}\right.}\right)}{\prod_{1}^{n}\left(s-c_{i}\right)^{n_{i}}}, \\
\sum_{1}^{l} l_{i}<\sum_{1}^{n} n_{i}=\sum_{1}^{m} m_{i}+p-1
\end{gathered}
$$

$$
R=2 p+2 n+2 \sum_{1}^{n} n_{i}+6-\lambda-2 \mu-\nu-\sum_{n-r+1}^{n} \rho_{i}
$$

and the order of $\Gamma$ is

$$
O=6 p+4 n+2 \sum_{1}^{n} n_{i}+6-2 \lambda-\lambda_{2}-4 \mu-3 \nu-\sum_{n-r+1}^{n} \sigma_{i} .
$$

If, however, $F(s)$ is defined by a binomial equation we must have

$$
\nu=\rho_{i}=\sigma_{i}=0
$$

and $\mu$ will denote the number of points $c_{i}=e_{i}$. 
The last part of the theorem follows by comparing the developments of

$$
F(s)=\frac{\prod_{1}^{m}\left(s-b_{i}\right)^{n_{i}} \sqrt{\prod_{1}^{2 p+2}\left(s-e_{i}\right)}}{\prod_{1}^{n}\left(s-c_{i}\right)^{n_{i}}}, \quad \sum_{1}^{n} n_{i}=\sum_{i_{i}}^{m} n_{i}+p-1
$$

with those given in $\S 5$; for $\mu$ and $\nu$ denote essentially the numbers of branch points about which the developments of $F(s)$ in series start with terms whose exponents are respectively $-\left(2 n_{i}-1\right) / 2$ and $-2 n_{i} / 2$; hence $\mu$ denotes in this case the number of points $c_{i}=e_{i}$ and $\nu=0$.

The application of that part of $\S 2$ which deals with $\chi(s)$ to the developments in $\S 5$ gives easily the result that

The class of $\Gamma$ is

$$
C=2 \sum_{1}^{n} n_{i}-\mu+4-\sum_{n-r+1}^{n} \tau_{i}
$$

where

$$
\tau_{i}=\left\{\begin{array}{ccc}
0 & \text { if conditions }(11) \text { are not satisfied, } \\
r_{i}+1 & \text { “ } & r_{i}<n_{i}-1 \\
n_{i} & \text { “ } & r_{i} \geqq n_{i}-1
\end{array}\right.
$$

\section{§7. Examples.}

To illustrate the results of the preceding section we will consider the minimum curves of lowest rank for which $F(s)$ is two-valued. All curves of given rank $R$ may be obtained by finding all integral solutions of the inequalities

$$
\left\{\begin{aligned}
2 p+2 n+2 \sum_{1}^{n} n_{i}+6-\lambda-2 \mu-\nu-\sum_{n-r+1}^{n} \rho_{i} & =R \\
\lambda+\mu+\nu & \leqq 2 p+2 \\
\mu+\nu+\nu & \leqq n
\end{aligned}\right.
$$

consistent with (9) and (13). It is not difficult to see that there are no solutions of these inequalities unless $R>4$. For we have

$$
\begin{gathered}
\lambda+\mu+\nu \leqq 2 p+2, \\
\mu+\sum_{n-r+1}^{n} \rho_{i} \leqq \sum_{n-r+1}^{n} n_{i}+r+\mu \leqq \sum_{1}^{n} n_{i}+n,
\end{gathered}
$$

hence, adding,$$
\lambda+2 \mu+\nu+\sum_{n-r+1}^{n} \rho_{i} \leqq 2 p+n+\sum_{1}^{n} n_{i}+2 .
$$

Trans. Am. Math. Soc. 31 
Therefore

$$
R \geqq n+\sum_{1}^{n} n_{i}+4,
$$

and hence $R>4$ unless $n=\sum n_{i}=0$; in the latter case

$$
\mu=\nu=\rho_{i}=0, \quad \sum m_{i}=1,
$$

and hence $\lambda \leqq 1$ which gives $R \geqq 5$.

We will now consider the three cases in which $R=5,6$ or 7 .

1. $R=5$. The inequalities (14) admit of the single solution

$$
p=n=n_{i}=\mu=\nu=\rho_{i}=0, \quad \lambda=1,
$$

which gives the function

$$
F(s)=\left(s-e_{1}\right) \sqrt{\left(s-e_{1}\right)\left(s-e_{2}\right)} .
$$

The corresponding minimum curve $\Gamma$ is of order 4 and class 4 . The equations of this curve, in two different positions, have been given by RICHмOND* in rational form. These positions of $\Gamma$ are obtained by rotating $\Gamma$ so that $F(s)$ is replaced by $s \sqrt{s}$ or $\left(s-e_{1}\right) \sqrt{\left(s-e_{1}\right) s}$; in the latter case RICHMOND's point of view gives a geometrical interpretation of the constant $\dot{e}_{1}$.

2. $R=6$. When $R=6$ there are two solutions of (14). The first is

$$
p=n=n_{i}=\mu=\nu=\rho_{i}=\lambda=0 .
$$

This solution gives

$$
F(s)=(s-b) \sqrt{\left(s-e_{1}\right)\left(s-e_{2}\right)},
$$

which reduces to the preceding case if $b=e_{1}$ or $e_{2} . \quad \Gamma$ is of order 6 and class 4 .

The second solution is

so that

$$
n=n_{1}=1, \quad p=\mu=\nu=0, \quad \lambda=\rho_{1}=2 .
$$

$$
\begin{gathered}
F(s)=\frac{C+\left(s-e_{1}\right)\left(s-e_{2}\right) \sqrt{\left(s-e_{1}\right)\left(s-e_{2}\right)}}{(s-c)}, \\
C=\left(c-e_{1}\right)^{\frac{3}{2}}\left(c-e_{2}\right)^{2} .
\end{gathered}
$$

The corresponding minimum curve is of order 5 and class 5 .

The preceding curves have been given by LIE, $\uparrow$ but his point of view did not enable him to give the functions $F(s)$ to which the curves correspond.

\footnotetext{
* On the Simplest Algebraic Minimal Curves, and the Derived Real Minimal Surfaces, Transactions of the Cambridge Philosophical Society, vol. 19 (1900), pp. 74, 75. In accordance with the theorem of Schwarz, Mathematische Abhandlungen, vol. 2, p. 22, the curves of rank 7 or less may be expressed rationally in terms of a parameter and hence we expect that there are no solutions of (14) for $R \equiv 7$ unless $p=0$.
}

†. .., p. 380 . 
3. $R=7$. There are three solutions of (14) when $R=7$. The first is

which gives

$$
n=n_{1}=1, \quad p=\mu=\nu=0, \quad \lambda=1, \quad \rho_{1}=2,
$$

$$
\begin{gathered}
F(s)=\frac{C+(s-b)\left(s-e_{1}\right) \sqrt{\left(s-e_{1}\right)\left(s-e_{2}\right)}}{s-c} . \\
C=(c-b)\left(c-e_{1}\right)^{s}\left(c-e_{2}\right)^{z} .
\end{gathered}
$$

The order of $\Gamma$ is 7 unless $b=e_{1}$ when the order reduces to 6 . In either case the class is 5 . If $b=e_{2}$ then $R$ reduces to 6 .

A second solution is

$$
n=1, \quad n_{1}=2, \quad p=\mu=\nu=0, \quad \lambda=2, \quad \rho_{1}=3,
$$

so that

$$
\begin{gathered}
F(s)=\frac{C(s-a)+(s-b)\left(s-e_{1}\right)\left(s-e_{2}\right) \sqrt{\left(s-e_{1}\right)\left(s-e_{2}\right)}}{(s-c)^{2}}, \\
C(c-a)=(c-b)\left(c-e_{1}\right)^{\frac{3}{2}}\left(c-e_{2}\right)^{\frac{3}{2}} \\
C \frac{d}{d s}[s-a]_{s=c}=\frac{d}{d s}\left[(s-b)\left(s-e_{1}\right)^{\frac{3}{2}}\left(s-e_{2}\right)^{\frac{3}{2}}\right]_{s=c},
\end{gathered}
$$

The order of $\Gamma$ is 6 unless $b=e_{1}$ or $e_{2}$ when the order is 5 ; its class is 6 .

Finally, there is a solution for $F(s)$ defined by a binomial quadratic ; viz.:

$$
p=0, \quad n=n_{1}=\mu=\lambda=1 .
$$

Hence

$$
F(s)=\frac{(s-b)\left(s-e_{1}\right) \sqrt{\left(s-e_{1}\right)\left(s-e_{2}\right)}}{\left(s-e_{2}\right)} .
$$

The order of the corresponding minimum curve is 6 or if $b=e_{1}$, it is 5 . The class is 5 .

In like manner we can determine all minimum curves for which $F(s)$ is twovalued of a given rank, order or class.

The examples which have been given all correspond to solutions of (14) of the form $p=0, n \leqq 1, n_{1} \leqq 2$; it is not difficult, although somewhat tedious, to show that there are no other solutions of this form if $R \leqq 7$. That all possible solutions for $R \leqq 7$ have been given will follow if it is shown that there are no solutions of the three forms

$$
\begin{aligned}
& \text { 1. } p=0, \quad n=1, \quad n_{1}>2 \text {, } \\
& \text { 2. } \quad p=0, \quad n>1, \quad \text { (hence, also, for } p>0, n>1 \text {.) } \\
& \text { 3. } p>0, \quad n \leqq 1 \text {. }
\end{aligned}
$$


The proof in cases 1 and 2 follows at once from the fact that

$$
R \geqq n+\sum_{1}^{n} n_{i}+4
$$

for in each case $n+\sum n_{i}>3$. In case 3 notice that

and hence

$$
\begin{gathered}
\mu+\sum_{n-r+1}^{n} \rho_{i} \leqq \sum_{1}^{n} n_{i}+n \\
\mu+\nu \leqq n
\end{gathered}
$$

Also

Hence

$$
2 \mu+\nu+\sum \rho_{i} \leqq 2 n+\sum n_{1} \cdot
$$

if $p>0$.

$$
\lambda \leqq m \leqq \sum m_{i}=\sum n_{i}-p+1 \text {. }
$$

$$
R \geqq 3 p+5 \geqq 8
$$

§ 8. Application to minimum surfaces.

In the paper already cited LIE has shown that on a minimum surface any two minimum curves of the same family are congruent and may be brought into coincidence by a translation. He then gives rules for the determination of the order and class of a minimum surface in terms of the rank and order of the minimum curves lying on the surface and the number of values the functions defining those curves take on for a given value of the argument. Hence the results of the preceding sections are immediately applicable to the determination of the order and class of algebraic minimum surfaces.

YaLE UNIVERSITY. 